\title{
The effect of hydrostatic pressure, temperature and magnetic field on the nonlinear optical properties of asymmetrical Gaussian potential quantum wells
}

\author{
Zhi-Hai Zhang ${ }^{1 *}$, LiLi Zou ${ }^{1}$, Kang-Xian Guo ${ }^{2}$, Jian-Hui Yuan ${ }^{3 * *}$ \\ ${ }^{1}$ School of New Energy and Electronic Engineering, Yancheng Teachers \\ University, Yancheng, 224051, China \\ ${ }^{2}$ Department of Physics, College of Physics and Electronic Engineering, Guangzhou \\ University, Guangzhou 510006, China \\ ${ }^{3}$ Department of Physics, Guangxi medical university, Nanning, Guangxi, 530021, China
}

\begin{abstract}
In this study, simultaneous effects of hydrostatic pressure, temperature and magnetic field on the linear and nonlinear intersubband optical absorption coefficients (OACs) and refractive index changes (RICs) in asymmetrical Gaussian potential quantum wells (QWs) are theoretically investigated within the framework of the compact-density-matrix approach and iterative method. The energy eigenvalues and their corresponding eigenfunctions of the system are calculated with the differential method. Our results show that the position and the magnitude of the resonant peaks of the nonlinear OACs and RICs depend strongly on the hydrostatic pressure, temperature and external magnetic field. This gives a new degree of freedom in various device applications based on the intersubband transitions of electrons.
\end{abstract}

Keywords: Optical properties; Quantum wells; Hydrostatic pressure; Temperature; Magnetic field.

\footnotetext{
${ }^{*}$ Corresponding author

${ }^{* *}$ Corresponding author

Email addresses: zhangzhihai3344@mail.bnu.edu.cn; Tel: 183-6114-8893 (Zhi-Hai Zhang ${ }^{1}$ ), jianhui831110@163.com; Tel:150-7882-3937 (Jian-Hui Yuan ${ }^{3}$ )
} 


\section{Introduction}

With recent rapid advances of modern technology, such as molecular beam epitaxy and metal-organic chemical vapour deposition [1, 2], it has become possible to produce a variety of dimensionality semiconductor nanostructures $[3,4,5]$, which offer a wide range of potential applications for optoelectronic devices. Some of these applications include semiconductor lasers[6], single-electron transistors[7], quantum computing[8], optical memories[9] and infrared photodetectors[10]. To fully understand and predict experimental phenomenon, the nonlinear optical properties in these semiconductor structures have been intensively studied theoretically. Especially, much attention has been paid to the larger-band-gap low-dimensional semiconductor QWs structure because they are more advantageous to grow, process, and fabricate into devices than are small-band-gap semiconductors.

Recently, there is a considerable interest in the optical phenomena based on semiconductor QWs nanostructure in the presence of some external perturbations, such as electric field, magnetic field, hydrostatic pressure and temperture. It is because its band gap can be tuned and thereby the change in property may be applied for the electro and opto-electronic devices. In particular, by means of the applied hydrostatic pressure one may generate indirect transitions in the energy-space. This transitions come from the appearance of the hydrostatic pressure-induced conduction band minimum at the $X$ point of the Brillouin zone of the materials that make up the potential barriers. F. Ungan et al. published in 2011, have investigated the effects of hydrostatic pressure and doping concentration on subband structure and optical transitions in modulation-doped $G a A s / A l_{x} G a_{1-x} A s$ QW. The obtained results show that by changing the doping concentration, the hydrostatic pressure and the structure parameters change further the separation between the subbands, which gives a new degree of freedom in device applications[11]. A. Hakimyfard et al. have studied the simultaneous effects of pressure and magnetic field on intersubband optical transitions in Pöschl-Teller QW[12]. $\dot{\mathrm{I}}$. Karabulut et al. have theoretically studied the simultaneous influences of applied electric field, magnetic field and hydrostatic pressure on the nonlinear optical rectification and OACs in $G a A s / A l_{x} G a_{1-x} A s$ asymmetric double QWs. They have given the optimizing nonlinear OACs using adjustable the geometry of the structure. Besides, the combination of hydrostatic pressure and electric field provide a way of tuning the position of the maxima and minima of the nonlinear optical properties[13]. H. Dakhlaoui et al. analyzed 
the intersubband transitions and OACs in GaAs QW under the effects of hydrostatic pressure, the position and the concentration of Si $\delta$-doped layer[14]. Oubram et al. investigated the hydrostatic pressure on intersubband transitions of n-type doped GaAs QW[15]. Majority of provided results indicate that the various factors such as temperature, applied electric and magnetic field, and hydrostatic pressure affect the electronic and optical properties of these semiconductor materials. Particularly, the manipulation of hydrostatic pressure leads to change in the confinement of electrons and in different transitions, which is very useful to fabricated optoelectronics devices.

In the present work, we present a numerical study of the linear and nonlinear OACs and RICs in an asymmetrical Gaussian potential QWs. In the section 2, the eigenfunctions and eigenenergies of electron states are obtained using finite difference method, and the analytical expression for the OACs and RICs are derived by means of the compact-density-matrix approach and an iterative method. In some investigation, the Gaussian shape QWs have been widely studied. But we find both energy and wavefunction for the lowlying state in this model are wrong to applied in these works[16, 17, 18]. Therefore, we have written a comment for pointing out the incorrect[19]. In the section 3, the numerical results and discussions are presented for asymmetrical Gaussian potential QWs under the simultaneous effects of hydrostatic pressure, temperature, applied magnetic field and the fixed applied electric field. Because of the electric field effect and structural parameters on the nonlinear optical properties in asymmetrical Gaussian potential QWs have been discussed in our previous paper[20]. A brief summary is given in section 4 .

\section{Theory}

The Hamiltonian for an electron confined in the asymmetrical Gaussian potential QWs under the influence of applied electric and magnetic and the hydrostatic pressure (electric field oriented in the growth direction and magnetic field perpendicularly to the growth direction). Within the effectivemass approximation, the Hamiltonian of this system can be written as[21]

$$
\left(-\frac{\hbar}{2} \frac{d}{d_{z}}\left[\frac{1}{m^{*}(P, T)} \frac{d}{d_{z}}\right]+V(z, P, T)+\frac{e^{2} B^{2}}{2 m^{*}(P, T) c^{2}}\right) \varphi(z)=E \varphi(z),
$$

where $z$ represents the growth direction of the QWs. $B$ is the external magnetic field, and $T$ is the temperature. $m^{*}(P, T)$ is the pressure- and 
temperature-dependent conduction effective mass in the well region, given by the expression[22, 23]

$$
\frac{m_{0}}{m^{*}(P, T)}=1+E_{p}^{\Gamma}\left[\frac{2}{E_{g}^{\Gamma}(P, T)}+\frac{1}{E_{g}^{\Gamma}(P, T)+\Delta_{0}}\right],
$$

where $m_{0}$ is the free electron mass, $E_{p}^{\Gamma}=7.51 \mathrm{eV}$ is the energy related to the momentum matrix element, $\Delta_{0}=0.341 \mathrm{eV}$ is the spin-orbit splitting, and $E_{g}^{\Gamma}(P, T)$ is the pressure- and temperature-dependent energy gap for the GaAs semiconductor at the $\Gamma$-point, given by[22]

$$
E_{g}^{\Gamma}(P, T)=E_{g}^{\Gamma}(0, T)+b P+c P^{2},
$$

where $E_{g}^{\Gamma}(0, T)=\left[1.519-\left(5.405 \times 10^{-4} T^{2}\right) /(T+204)\right] \mathrm{eV}, b=1.26 \times 10^{-2}$ $\mathrm{eV} / \mathrm{kbar}$ and $c=-3.77 \times 10^{-5} \mathrm{eV} / \mathrm{kbar}^{2}$.

And the confinement potential $V(z, P, T)$ is given by

$$
V(z, P, T)= \begin{cases}-V_{0}(P, T) \exp \left(-z^{2} / 2 L(P)^{2}\right) & z \geq 0 \\ \infty & z<0,\end{cases}
$$

here the barrier height is given by[24]

$$
V_{0}(p, T)=Q_{c} \Delta E_{g}^{\Gamma}(X, P, T),
$$

where $Q_{c}=0.6$ is the conduction band offset parameter, $X=0.3$ is the mole fraction of aluminum in $G a_{1-x} A l_{x} A s$, and $\Delta E_{g}^{\Gamma}(X, P, T)$ is the band gap difference between QW and barrier matrix at the $\Gamma$-point as a funtion of $P$ and $T$, which is given by[25]

$$
\Delta E_{g}^{\Gamma}(X, P, T)=\Delta E_{g}^{\Gamma}(X)+P D(X)+G(X) T,
$$

where $\Delta E_{g}^{\Gamma}(X)=\left(1.55 X+0.37 X^{2}\right) \mathrm{eV}$ the variation of the gap is difference and $D(X)=\left[-\left(1.3 \times 10^{-3}\right) X\right] \mathrm{eV} / \mathrm{kbar}, G(X)=\left[-\left(1.11 \times 10^{-4}\right) X\right] \mathrm{eV} / \mathrm{K}$. The pressure-dependent width of the QWs and barrier layer is given by

$$
L(P)=L(0)\left(1-\left(S_{11}+2 S_{12}\right) P\right) .
$$

where $S_{11}=1.16 \times 10^{-3} \mathrm{kbar}^{-1}$ and $S_{12}=3.7 \times 10^{-4} \mathrm{kbar}^{-1}$ are the elastic constants of the GaAs and $L(0)$ is the original width of the layers.

Due to the presence of the applied electric and magnetic fields and hydrostatic pressure in the Hamiltonian(Eq.(1)), it is impossible to find self-energy 
analytic eigenfunctions that correspond to the exact solution of one electron confined in asymmetrical Gaussian potential QWs. To obtain the eigenvalues and eigenvectors of the time-independent Schrödinger equation, we have adopted the finite difference method. Next we will derive the OACs and RICs in asymmetrical Gaussian potential QWs by the compact-densitymatrix method and the iterative procedure. The analytical expressions of the linear and the third-order nonlinear susceptibilities for a two-level quantum system are given as follows[26, 27, 28]. First, for the linear term,

$$
\varepsilon_{0} \chi^{(1)}(\omega)=\frac{N\left|M_{21}\right|^{2}}{E_{21}-\hbar \omega-i \hbar \Gamma_{12}} .
$$

For the third-order term,

$$
\begin{aligned}
\varepsilon_{0} \chi^{(3)}(\omega)= & -\frac{N\left|M_{21}\right|^{2}}{E_{21}-\hbar \omega-i \hbar \Gamma_{12}} \\
& \times\left[\frac{4\left|M_{21}\right|^{2}}{\left(E_{21}-\hbar \omega\right)^{2}+(\hbar \omega)^{2}}\right. \\
& \left.-\frac{\left(M_{22}-M_{11}\right)^{2}}{\left(E_{21}-\hbar \omega-i \hbar \Gamma_{12}\right)}\right] .
\end{aligned}
$$

The susceptibility $\chi(\omega)$ is related to the change in the refractive index as follow:

$$
\frac{\Delta n(\omega)}{n_{r}}=R e \frac{\chi(w)}{2 n_{r}^{2}},
$$

where $n_{r}$ is the refractive index. By using Eqs.(8-10), the linear and the third-order nonlinear refractive index changes are obtained by

$$
\frac{\Delta n^{(1)}(\omega)}{n_{r}}=\frac{1}{2 n_{r}^{2} \varepsilon_{0}}\left|M_{21}\right|^{2} N\left[\frac{E_{21}-\hbar \omega}{\left(E_{21}-\hbar \omega\right)^{2}+\left(\hbar \Gamma_{12}\right)^{2}}\right]
$$

and

$$
\begin{aligned}
\frac{\Delta n^{(3)}(\omega)}{n_{r}}= & -\frac{\mu c}{4 n_{r}^{3} \varepsilon_{0}}\left|M_{21}\right|^{2}\left[\frac{N I}{E_{21}-\hbar \omega}\left(E_{21}-\hbar \omega\right)^{2}+\left(\hbar \Gamma_{12}\right)^{2}\right]^{2} \\
& \times\left[4\left(E_{21}-\hbar \omega\right)\left|M_{21}\right|^{2}-\frac{\left(M_{22}-M_{11}\right)^{2}}{\left(E_{21}\right)^{2}+\left(\hbar \Gamma_{12}\right)^{2}}\left\{\left(E_{21}-\hbar \omega\right)\right.\right. \\
& \left.\left.\times\left[E_{21}\left(E_{21}-\hbar \omega\right)-\left(\hbar \Gamma_{12}\right)^{2}\right]-\left(\hbar \Gamma_{12}\right)^{2}\left(2 E_{21}-\hbar \omega\right)\right\}\right],
\end{aligned}
$$


where $N$ is the carrier density in this system, $\mu$ is the permeability of the system, $E_{i j}=E_{i}-E_{j}$ is the energy interval of two different electronic states, $M_{i j}$ is the matrix elements which is defined by $M_{i j}=\left|\left\langle\varphi_{i}|e x| \varphi_{j}\right\rangle\right|(i, j=1,2)$. $I$ is the incident optical intensity and defined as

$$
I=2 \sqrt{\frac{\varepsilon_{R}}{\mu}}|E(\omega)|^{2}=\frac{2 n_{r}}{\mu c}|E(\omega)|^{2},
$$

where $c$ is the speed of light in free space. Therefore, the total refractive index change can be written as

$$
\frac{\Delta n(\omega)}{n_{r}}=\frac{\Delta n^{(1)}(\omega)}{n_{r}}+\frac{\Delta n^{(3)}(\omega)}{n_{r}} .
$$

In addition, the susceptibility $\chi(\omega)$ is related to the absorption coefficient $\alpha(\omega)$ by

$$
\begin{aligned}
& \alpha^{(1)}(\omega)=\omega \sqrt{\frac{\mu}{\varepsilon_{R}}} \frac{\left|M_{21}\right|^{2} N \hbar \Gamma_{12}}{\left(E_{21}-\hbar \omega\right)^{2}+\left(\hbar \Gamma_{12}\right)^{2}} . \\
& \alpha^{(3)}(\omega, I)=-\omega \sqrt{\frac{\mu}{\varepsilon_{R}}}\left(\frac{I}{2 \varepsilon_{0} n_{r} c}\right) \frac{\left|M_{21}\right|^{2} N \hbar \Gamma_{12}}{\left[\left(E_{21}-\hbar \omega\right)^{2}+\left(\hbar \Gamma_{12}\right)^{2}\right]^{2}}\left[4\left|M_{21}\right|^{2}\right. \\
&\left.-\frac{\left|M_{22}-M_{11}\right|^{2}\left[3 E_{21}^{2}-4 E_{21} \hbar \omega+\hbar^{2}\left(\omega^{2}-\Gamma_{12}^{2}\right)\right]}{E_{21}^{2}+\left(\hbar \Gamma_{12}\right)^{2}}\right] .
\end{aligned}
$$

So, the total absorption coefficient $\alpha(\omega, I)$ is given by

$$
\alpha(\omega, I)=\alpha^{(1)}(\omega)+\alpha^{(3)}(\omega) .
$$

\section{Results and discussions}

In this section, we will discuss the optical absorption coefficient and refractive index changes in asymmetrical Gaussian potential QWs under the influence of combined independent external electric and magnetic fields as well as hydrostatic pressure. The parameter adopted in our calculation are as follows [29, 30]: $N=5 \times 10^{16} \mathrm{~cm}^{-3}, n_{r}=3.2, T_{12}=0.14 \mathrm{ps}, \Gamma_{12}=1 / T_{12}$, $I=1.0 \mathrm{MW} / \mathrm{cm}^{2}$.

In Fig.1, the variations of the confinement potential profile is plotted as a function the position $z$ for different values of the applied magnetic field, hydrostatic pressure and temperature. Fig.1(a) and (b) show that the changes 
of the well depth is clearly visible, where the bottom of the QWs shifts upward with increasing the hydrostatic pressure $P$ and temperature $T$. But the well width has a very small variation with the hydrostatic pressure $P$ and temperature $T$. Fig.1(c) shows that the well width reduces with the enhancement of the applied magnetic field $B$, which leads to the enhancement of quantum confinement, hence the ground state and the first excited state energy increase.

In Fig.2, the linear $\alpha^{(1)}(\omega)$, the third-order nonlinear $\alpha^{(3)}(\omega)$, and the total OACs $\alpha(\omega)$ are plotted as a function of the incident photon energy for three different values of $P($ a), the first two energy levels, the energy difference $E_{21}$ between ground states $E_{1}$ and first excited states $E_{2}(\mathrm{~b})$ and the resonant peak value of the total OACs $\alpha(\omega)$ (c) are depicted versus the hydrostatic pressure $P$ with $B=0 \mathrm{~T}, T=0 \mathrm{~K}, L=4 \mathrm{~nm}$. From Fig.2(a), we find that (1) the magnitude of the linear absorption coefficient is significantly reduced with the increasing of the hydrostatic pressure $P$. And the magnitude of the nonlinear absorption coefficient decreases slightly. Moreover, the large linear change generated by the $\chi^{(1)}$ term is opposite in sign of the nonlinear change generated by the $\chi^{(3)}$ term. The total absorption coefficient is increased by the linear term, but it is significantly reduced by the third-order nonlinear term. Therefore, the total OACs are decreasing as the hydrostatic pressure $P$ increases. (2) the linear, the third-order nonlinear and the total OACs have a maximum value where photon energy equal to the energy difference $E_{21}$ between ground states $E_{1}$ and first excited states $E_{2}$, which is the resonance photon energy. (3) the resonant peak positions have an obvious red-shift with the hydrostatic pressure $P$ increases, which can be interpreted by the variations of the energy difference $E_{21}$ between ground states $E_{1}$ and first excited states $E_{2}$ with varying the hydrostatic pressure $P$ (see in Fig.2(b)). As seen in Fig.2(b), the energy difference $E_{21}$ between the two different electronic states decreased as the hydrostatic pressure $P$ increases, which will lead to the resonance peak red-shift. To look at these behavior accurately, in Fig.2(c) the resonant peak value of the total OACs is plotted as a function of the hydrostatic pressure $P$. As the hydrostatic pressure $P$ increases, the resonant peak of the total OACs gradually decreases. The physical reason is that the hydrostatic pressure has an effect similar to reducing the confinement, which increases the electrons effective mass, and this in turn results in a decrease of the strength confinement[31].

In Fig.3, the linear $\alpha^{(1)}(\omega)$, the third-order nonlinear $\alpha^{(3)}(\omega)$, and the total OACs $\alpha(\omega)$ are plotted as a function of the incident photon energy for 
three different values of $T$ (a), the first two energy levels, the energy difference $E_{21}$ between ground states $E_{1}$ and first excited states $E_{2}(\mathrm{~b})$ and the resonant peak value of the total OACs $\alpha(\omega)(c)$ are depicted versus the temperature $T$ with $B=0 \mathrm{~T}, P=0 \mathrm{kbar}, L=4 \mathrm{~nm}$. From Fig.3(a), it can be clearly seen that the resonant peaks of the linear, the third-order nonlinear and the total OACs experience a slight red-shift with the increase in the temperature $T$. It is due to the decrease in energy difference between ground state and first excited state(see in Fig.3(b)). From Fig.3(b), the ground state increases slightly with the increasing of the temperature $T$. However, the first excited state hardly changed. So the energy difference $E_{21}$ between the two different electronic states decreases slightly when the temperature $T$ increases. In addition, the magnitude of the linear and the third-order nonlinear OACs increase simultaneously with the increase of the temperature $T$, while based on the opposite sign of linear and nonlinear terms, the total OACs will behave similar to the major term. From Fig.3(a) and (b), it is obvious that the major term is linear term, so the total OACs will be like linear term. Behavior of the total OACs is shown versus the temperature $T$ in Fig.3(c). This figure shows that with the increase of the temperature $T$, the resonant peak value of the total OACs increases.

the linear $\alpha^{(1)}(\omega)$, the third-order nonlinear $\alpha^{(3)}(\omega)$, and the total OACs $\alpha(\omega)$ as a function of the incident photon energy with $P=0 \mathrm{kbar}, T=0$ $\mathrm{K}, L=4 \mathrm{~nm}$ are shown in Fig.4(a). Several distinct values of the applied magnetic field: $B=0 \mathrm{~T}, B=15 \mathrm{~T}$ and $B=30 \mathrm{~T}$ are taken into account, as it may be seen from the different curves presented. We noted that the applied magnetic field $B$ have a great influence on the OACs. First, the magnitude of the linear and the magnitude of the third-order nonlinear OACs increases as the applied magnetic field $B$ increases. And the magnitude of the total OACs increases as the applied magnetic field $B$ increases. This is because the linear term makes larger contribution to the total OACs. The other property is the resonant peak positions have an obvious blue-shift with the applied magnetic field $B$ increases. These features can be understood easily as follows: the well width reduces with the enhancement of the applied magnetic field $B$ (see Fig.1(c)), which leads to the stronger quantum confinement, so the energy difference $E_{21}$ between the first excited state and the ground state of the system increases, and also the corresponding peak position shifts toward higher energies (see Fig.4(b)). In addition, to show the relation between the OACs and applied magnetic field $B$ more clearly, we have plotted Fig.4(c), showing the variation of resonant peak of the total OACs as a function of 
the applied magnetic field $B$. It is observed that the resonant peak value of the total OACs $\alpha_{\max }$ significantly increases with applied magnetic field $B$ strengths.

In Fig.5, we present the variation of resonant peak value of the linear $\left|\Delta n^{(1)} / n_{r}\right|$, the third-order nonlinear $\left|\Delta n^{(3)} / n_{r}\right|$, and the total $\left|\Delta n / n_{r}\right|$ RICs as a function of the hydrostatic pressure $P$ (a), temperature $T$ (b) and applied magnetic field $B(\mathrm{c})$, respectively. The calculated parameters corresponding to the Fig.2(c), Fig.3(c) and Fig.4(c), respectively. As can seen from Fig.5(a), the resonant peak value of the linear term decreases by an increase in the hydrostatic pressure $P$. And the resonant peak value of the third-order nonlinear term increases slowly. However, the resonant peak of the total RICs will be reduced. This is because the sign of the linear and nonlinear RI changes is opposite, and the total RICs will behave similar to the linear term. From Fig.5(b), one should also note that the resonant peak value of the linear(nonlinear) OACs increases(decreases) as the temperature $T$ increases. And the resonant peak value of the total OACs increases with increasing temperature $T$. It is because of the linear term is the major term, and the total OACs behaves like the linear term. It is surprise to us that the resonant peak value of the total RICs do not monotonously(see the inset in Fig.5(c)). The resonant peak value of the total RICs increases as the applied magnetic field $B$ increases for the applied magnetic field $B<15 \mathrm{~T}$, which will decreases for the applied magnetic field $B>15 \mathrm{~T}$. The physical reason of this behavior is that the effect of magnetic field is more prominent for wider QWs. However, as the applied magnetic field $B$ goes up, the width of QWs reduces, thus the effect of confined will play a more important role.

In Fig.6(a) and (b) the combined effects of hydrostatic pressure $P$, temperature $T$ and applied magnetic field $B$ are considered in the calculation of the intensity of the resonant peak for the total OACs and RICs in asymmetrical Gaussian potential QWs with $L=4 \mathrm{~nm}$. From Fig.6(a), it may be noted that the intensity of the resonant peak for the total OACs tend to augment with the increase in temperature $T$ and applied magnetic field $B$. As it was previously mentioned, in this situation the confinement width of QWs is changed as a result of the increase in applied magnetic field $B$, and the electron effective mass is a decreasing function of the temperature $T$. So, the peak amplitude to augment is associated with the increase in temperature $T$ and applied magnetic field $B$. Accordingly, it is observed that the intensity of the peaks of resonance is decreased with increasing hydrostatic pressure $P$. This is a direct consequence of the fact that there is an increment in 
the electron effective mass as long as the value of the hydrostatic pressure $P$ in the system is augmented. In Fig.6(b) it is seen that the intensity of the resonant peak for the total RICs decreases by an increase in hydrostatic pressure $P$. The intensity of the resonant peak for the total RICs increases as the increasing temperature $T$. One should also note that variation of the resonant peak value of the total RICs is not monotonous for increasing applied magnetic field $B$ T.

\section{Conclusions}

In this paper, we investigated the intersubband OACs and the RICs in asymmetrical Gaussian potential QWs. We obtained the energy eigenvalues and the energy eigenfunctions by the finite different method. Numerical calculations show that the OACs and RICs depend dramatically on the hydrostatic pressure, temperature and the applied magnetic field. It was found that the applied magnetic field blue shifts the absorption peak, both linear and the third-order nonlinear terms, whereas the hydrostatic pressure and the temperature shift these peaks into the red end. Simultaneously, the influence of the temperature and the applied magnetic field cause an increase in the intensity of the resonant peak for the total OACs, whilst the intensity of the resonant peak for the total OACs is decreased by the hydrostatic pressure. The influence of the application of applied magnetic field is significant with respect to the effect of hydrostatic pressure and temperature, which determines the asymptotic tendencies observed in the OACs. The effect of hydrostatic pressure and temperature on the RICs is similar to OACs. But variation of the resonant peak value of the total RICs is not monotonous for increasing applied magnetic field. We hope that this theoretical investigation may have consequences about practical application of electro-optical devices and optical communication.

Acknowledgments: Project supported by the National Science Fundation of China (under Grant No. 11447193, 11447101, 61178003 and 61475039), the Youth Science Foundation of Guangxi Medical University in China (under Grant No. GXMUYSF201313), the Guangxi Department of Education Research Projects in China (under Grant No. KY2015LX046) and the University National Science Foundation of Jiangsu Province of China (under Grant No. 14KJB140015). 


\section{References}

[1] P.D. Dapkus, Annu, Rev. Mater. Sci. 12 (1982) 243.

[2] S. Hersee, J.P. Duchemim, Annu, Rev. Mater. Sci. 12 (1982) 65.

[3] M. Sundaram, S.A. Chalmers, P.E. Hopkins, A.C. Gossard, Science 254 (1991) 1326.

[4] M.R. Panish, Science 208 (1980) 916.

[5] C. Sirtori, F. Capasso, D.L. Sivco, A.Y. Cho, Phys. Rev. Lett. 68 (1992) 1010 .

[6] N. Kristaedter, O.G. Schmidt, N.N. Ledentsov, D. Bimberg, V.M. Ustinov, A.Yu. Egorov, A.E. Zhukov, M.V. Maximov, P.S. Kopev, Zh.I. Alferov, Appl. Phys. Lett. 69 (1996) 1226.

[7] E. Leobandung, L.J. Guo, S.Y. Chou, Appl. Phys. Lett. 67 (1995) 2338.

[8] D. Loss, D.P. Divicenzo, Phys. Rev. A 57 (1998) 120.

[9] K. Imamura, Y. Sugiyama, Y. Nakata, N. Muto, N. Yokoyama, Jpn. J. Appl. Phys. 34 (1995) L1445.

[10] X. Jiang, S.S. Li, M.Z. Tidrow, Physica E 5 (1999) 27.

[11] F. Ungan, E. Kasapoglu, I. Sokmen, Solid State Commun. 151 (2011) 1415.

[12] A. Hakimyfard, M.G. Barseghyan, A.A. Kirakosyan, Physica E 41 (2009) 1596.

[13] İ. Karabulut, M.E. Mora-Ramos, C.A. Duque, J. Lumin. 131 (2011) 1502.

[14] H. Dakhlaoui, and S. Almansour, E. Algrafy, Superlatt. Microstruct. 77 (2015) 196.

[15] O. Oubram, O. Navarro, L.M. Gaggero-Sager, J.C. Martínez-Orozco, I. Rodríguez-Vargas, Solid State Commun. 14 (2012) 440.

[16] A.X. Guo, J.F. Du, Superlatt. and Microstruct. 64 (2013) 158-166. 
[17] J.H. Wu, K.X. Guo, G.H. Liu, Physica B 446 (2014) 59-62.

[18] W.J. Zhai, Physica B 454 (2014) 50-55.

[19] J.H. Yuan, Z.H. Zhang, Superlatt. Microstruct. doi:10.1016/j.spmi.2015.04.001.

[20] Z.H. Zhang, L.L. Zou, C.L. Liu, J.H. Yuan, Superlatt. Microstruct. 85 (2015) 385.

[21] N. Eseanu, E.C. Niculescu, L.M. Burileanu, Phys. E 41 (2009) 1386.

[22] H. Ehrenrich, J. Appl. Phys. 32 (1961) 2155.

[23] D.E. Aspnes, Phys. Rev. B 14 (1976) 5331.

[24] R.F. Kopf, M.H. Herman, M.L. Schnoes, A.P. Perley, G. Livescu, M. Ohring, J. Appl. Phys. 71 (1992) 5004.

[25] A. Montes, A.L. Morales, C.A. Duque, Surf. Rev. Lett. 9 (2002) 1753.

[26] S. Ünlü, İ. Karabulut, H. Safak, Physica. E 33 (2006) 319.

[27] C.H. Liu, B.R. Xu. Phys. Lett. A 33 (2006) 324.

[28] Y.B. Yu, S.N. Zhu, K.X. Guo, Solid State Commun. 139 (2006) 76.

[29] K.X. Guo, C.Y. Chen, Physica B 269 (1999) 139.

[30] Z.H. Zhang, K.X. Guo, B. Chen, R.Z. Wang, M.W. Kang, S. Shao, Superlatt. Microstruct. 47 (2010) 325.

[31] L. Bouzaiene, R. Ben Mahrsia, M. Baira, L. Sfaxi, H. Maaref, J. Lumin. 135 (2013) 271. 


\section{Figure Captions}

Figure 1 The variation of the confinement potential profile are depicted as a function of the position $\mathrm{z}$ for different values of (a)the hydrostatic pressure $P$ with $T=0 \mathrm{~K}, B=0 \mathrm{~T}, L=4 \mathrm{~nm}$, (b)temperature $T$ with $P=0 \mathrm{kbar}$, $B=0 \mathrm{~T}, L=4 \mathrm{~nm}$, and (c)applied magnetic field $B$ with $P=0 \mathrm{kbar}, T=0$ $\mathrm{K}, L=4 \mathrm{~nm}$.

Figure 2 The linear $\alpha^{(1)}(\omega)$, the third-order nonlinear $\alpha^{(3)}(\omega)$ and the total OACs $\alpha(\omega)$ versus the incident photon energy with three different strength of the hydrostatic pressure $P=0,50$, and $100 \mathrm{kbar}(\mathrm{a})$, the low-lying energy levels of $E_{1}, E_{2}$, the difference $E_{21}$ (b) and the intensity of the total OACs peak of resonance(c) versus the hydrostatic pressure for $B=0 \mathrm{~T}, T=0 \mathrm{~K}$, $L=4 \mathrm{~nm}$.

Figure 3 The linear $\alpha^{(1)}(\omega)$, the third-order nonlinear $\alpha^{(3)}(\omega)$ and the total OACs $\alpha(\omega)$ versus the incident photon energy with three different strength of the temperature $T=0,200$, and $400 \mathrm{~K}(\mathrm{a})$, the low-lying energy levels of $E_{1}, E_{2}$, the difference $E_{21}(\mathrm{~b})$ and the intensity of the total OACs peak of resonance(c) versus the temperature for $B=0 \mathrm{~T}, P=0 \mathrm{kbar}, L=4 \mathrm{~nm}$.

Figure 4 The linear $\alpha^{(1)}(\omega)$, the third-order nonlinear $\alpha^{(3)}(\omega)$ and the total OACs $\alpha(\omega)$ versus the incident photon energy with three different strength of the magnetic field $B=0,15$, and $30 \mathrm{~T}(\mathrm{a})$, the low-lying energy levels of $E_{1}, E_{2}$, the difference $E_{21}(\mathrm{~b})$ and the intensity of the total OACs peak of resonance(c) versus the applied magnetic field for $P=0 \mathrm{kbar}, T=0 \mathrm{~K}$, $L=4 \mathrm{~nm}$.

Figure 5 The peak of the total RICs $\left|\Delta / n_{r}\right|$ versus (a)the hydrostatic pressure for $B=0 \mathrm{~T}, T=0 \mathrm{~K}, L=4 \mathrm{~nm}$, (b)the temperature for $B=0$ $\mathrm{T}, P=0 \mathrm{kbar}, L=4 \mathrm{~nm}$, and (c)the applied magnetic field for $P=0 \mathrm{kbar}$, $T=0 \mathrm{~K}, L=4 \mathrm{~nm}$.

Figure 6 Variations of (a)the total OACs $\alpha$ and (b)the total RICs $\left|\Delta / n_{r}\right|$ with the combined effect of the hydrostatic pressure, temperature and applied magnetic field. 
Fig.1

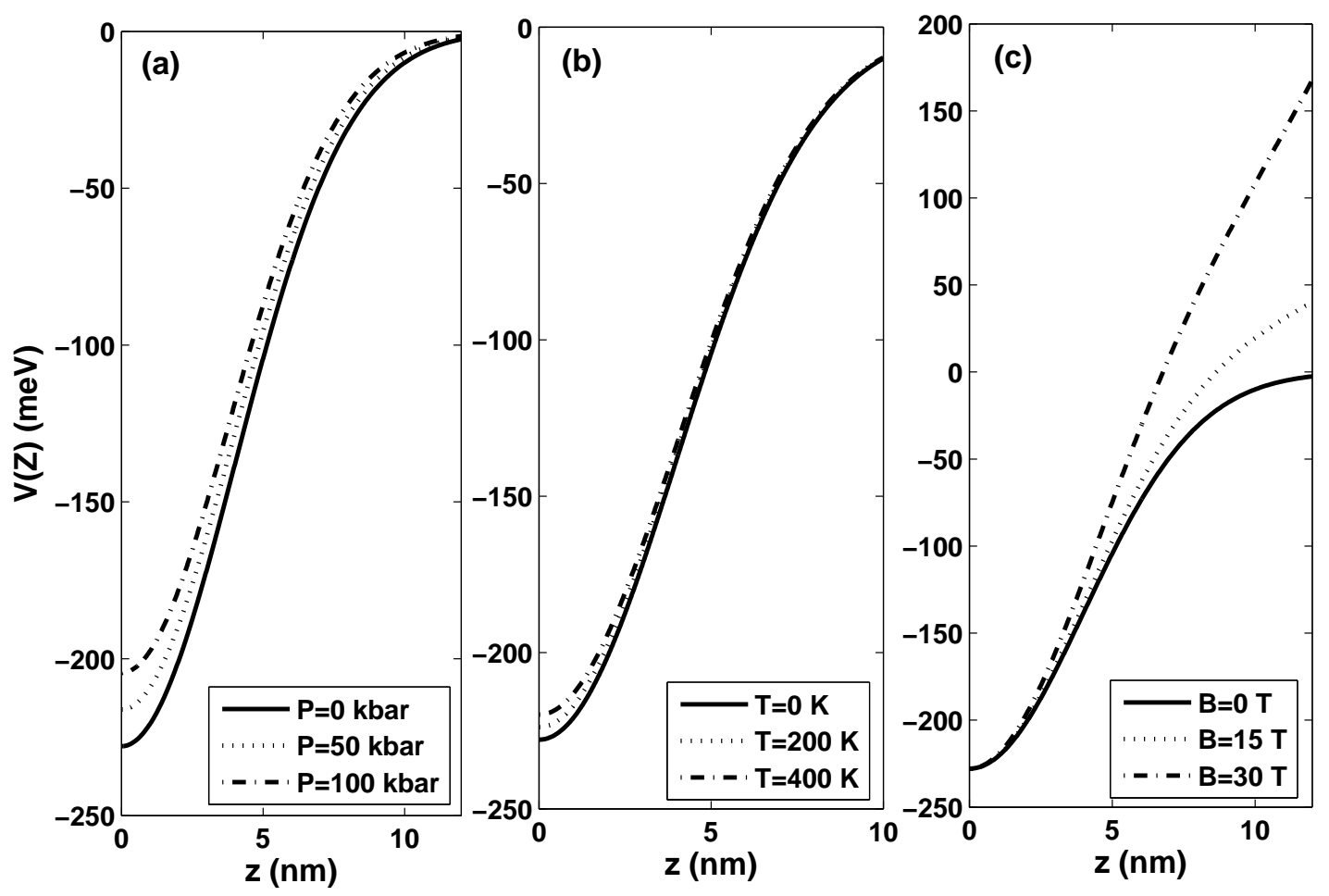




\section{Fig.2}
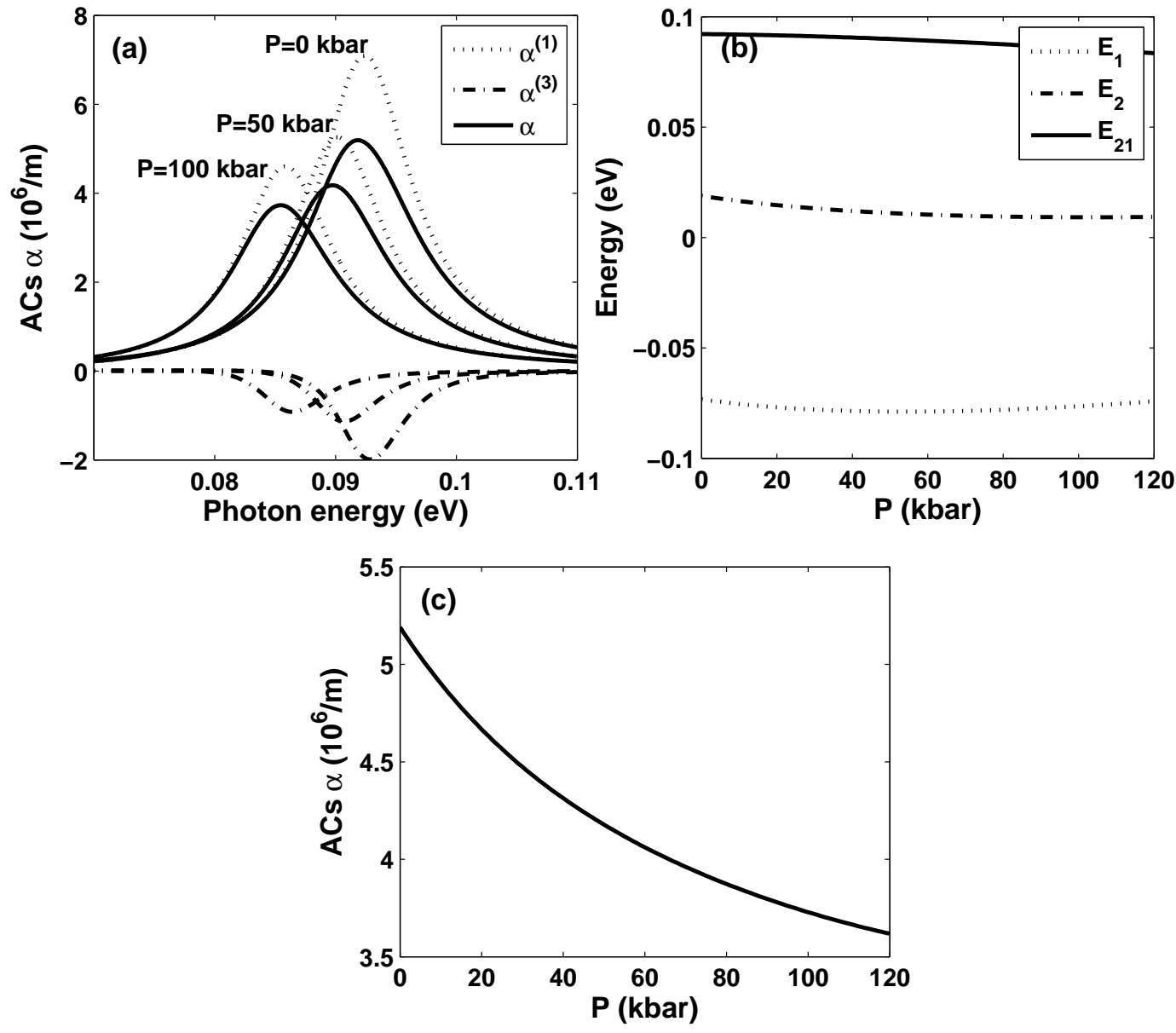
Fig.3
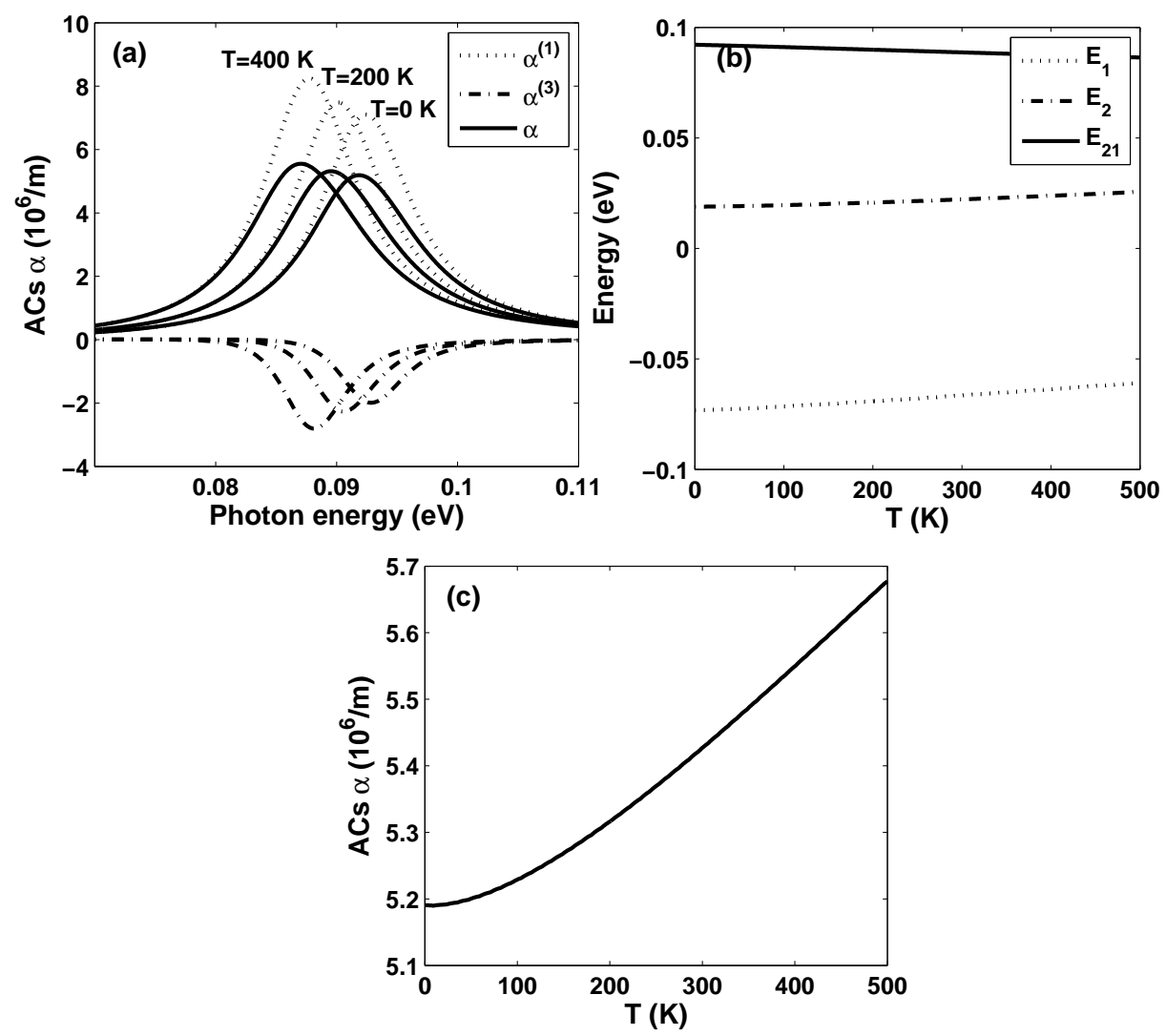
Fig.4
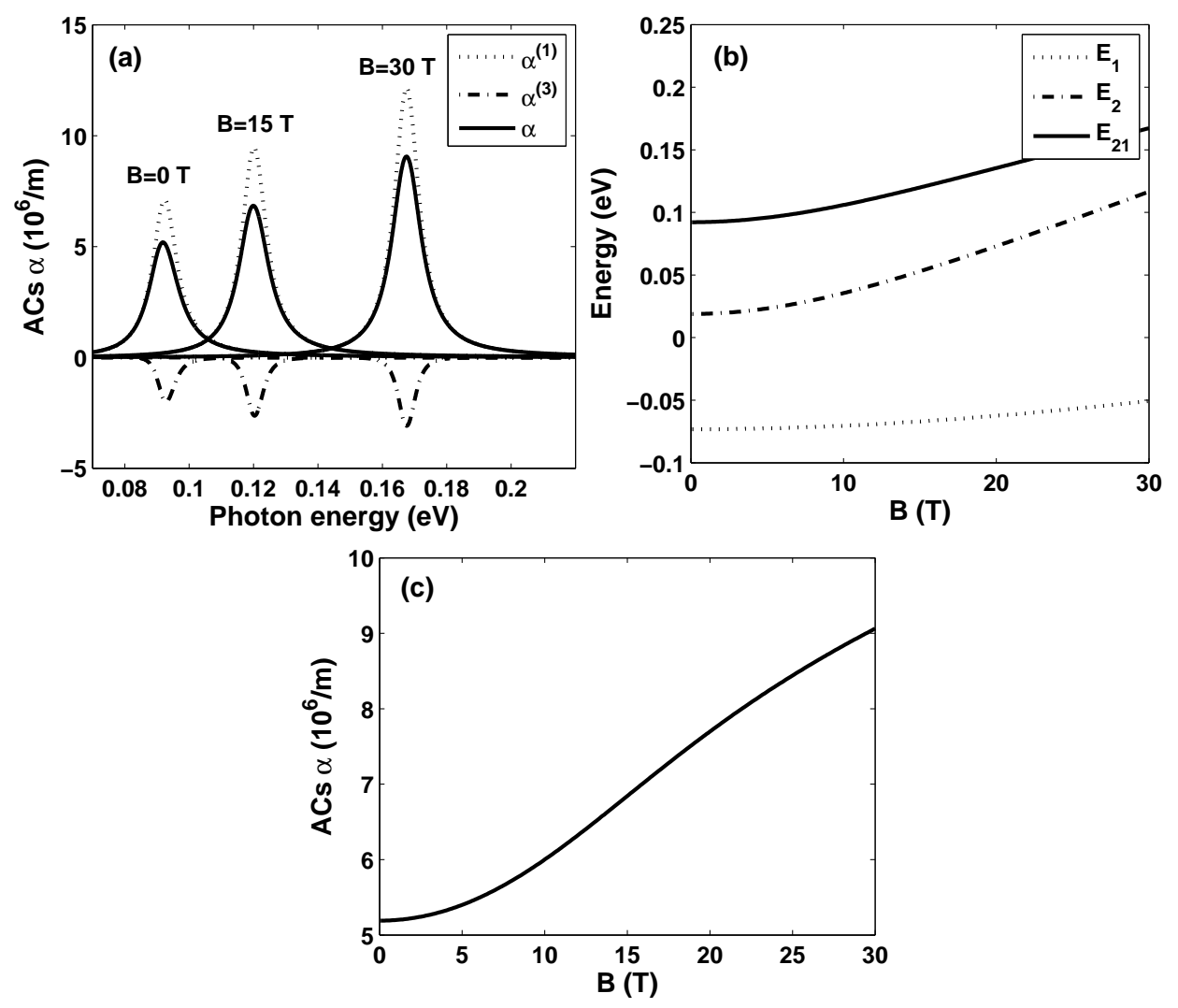
Fig.5

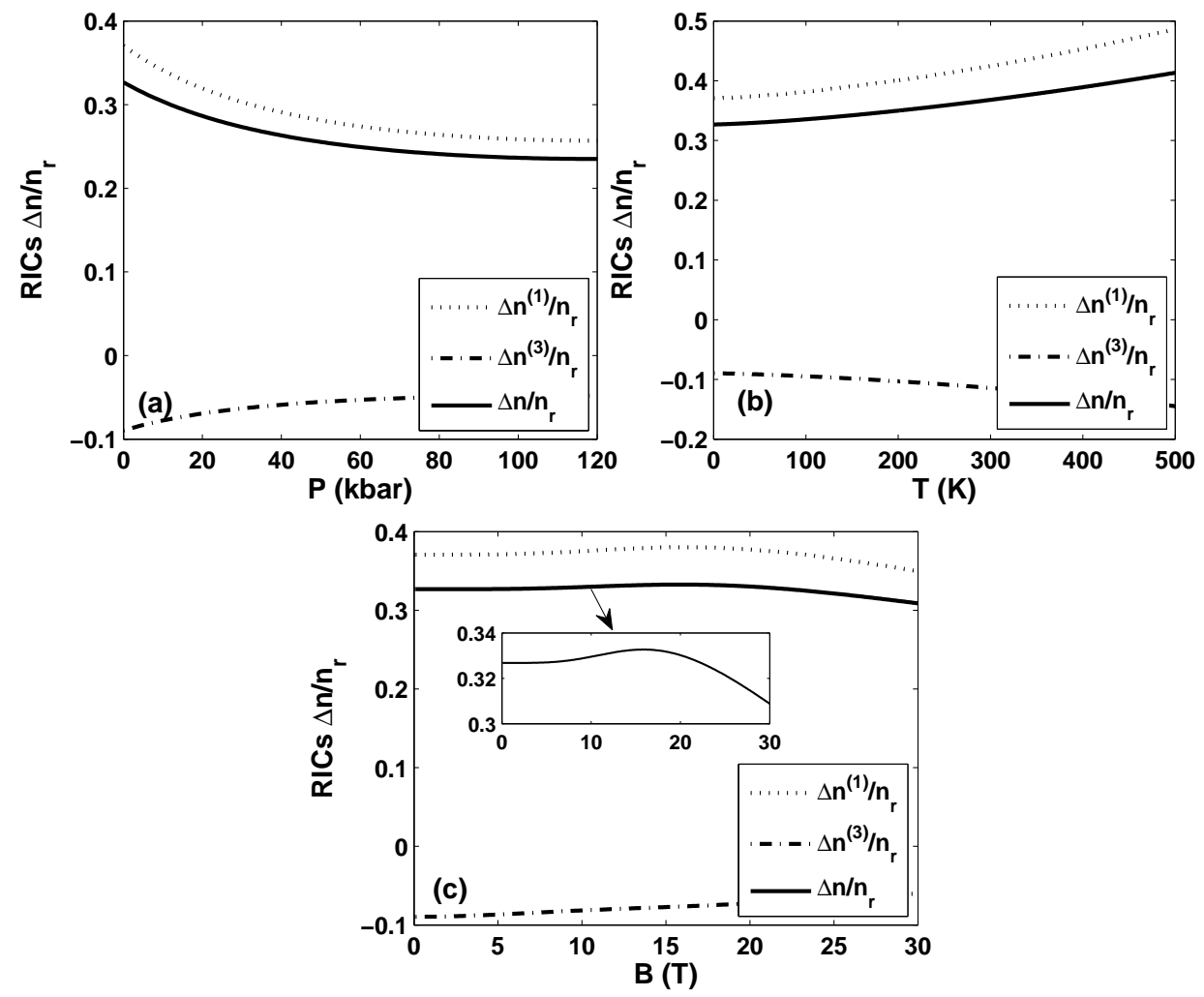


Fig.6
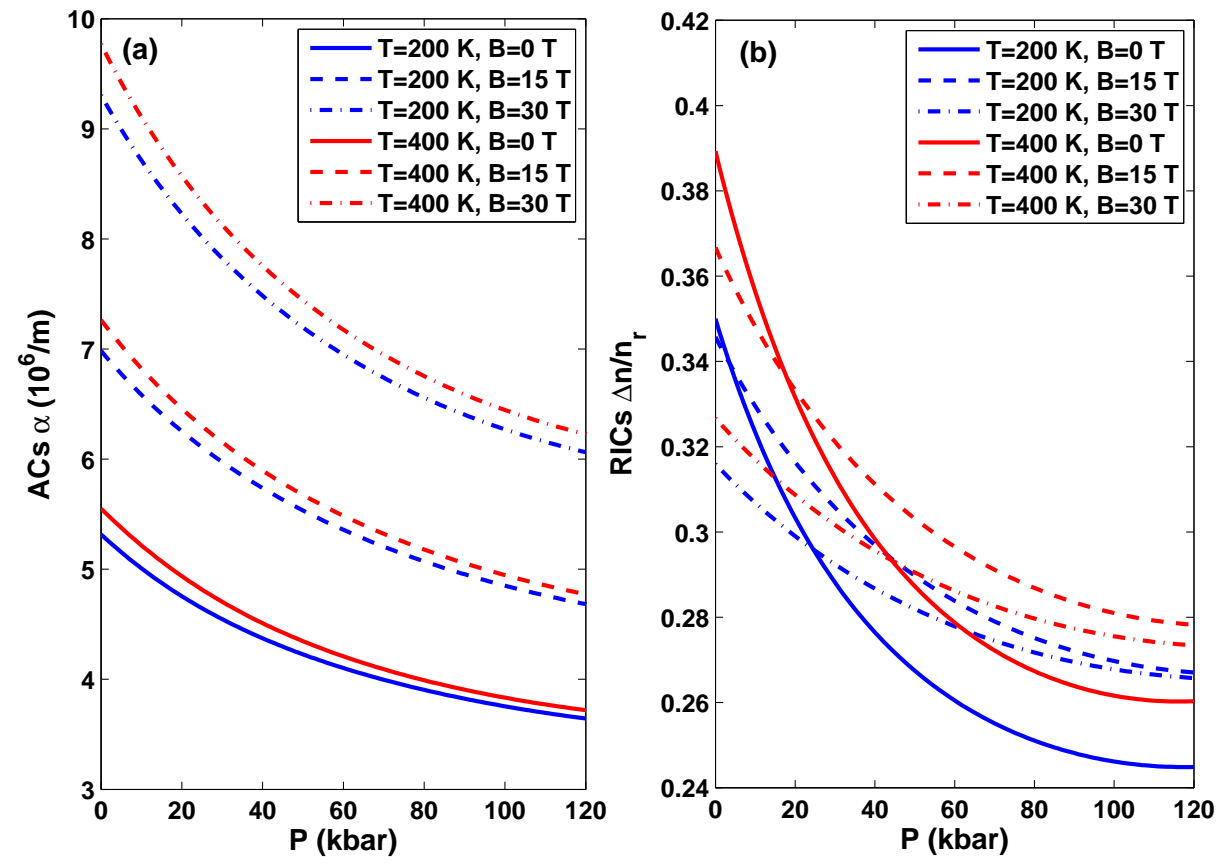